Musées, Patrimoine et Culture scientifiques et techniques

$124 \mid 2009$

juillet - août 2009

\title{
De la domination et de la soumission : la sujétion du musée à l'autorité de l'État
}

\section{François Cheval}

\section{(Q) OpenEdition \\ Journals}

Édition électronique

URL : http://journals.openedition.org/ocim/299

DOI : 10.4000/ocim.299

ISSN : 2108-646X

Éditeur

OCIM

Édition imprimée

Date de publication : 1 juillet 2009

Pagination : 19-24

ISSN : 0994-1908

Référence électronique

François Cheval, « De la domination et de la soumission : la sujétion du musée à l'autorité de l'État », La Lettre de I'OCIM [En ligne], 124 | 2009, mis en ligne le 01 juillet 2011, consulté le 30 avril 2019. URL http://journals.openedition.org/ocim/299; DOI : 10.4000/ocim.299 
universitaire. Quand, sous cette forme, au début des années 1980, il est apparu évident que les musées erraient dans le monde contemporain, que l'institution avait failli comme entreprise de diffusion de savoir, la réponse apportée fut de les multiplier. Le pouvoir politique, porté par un mouvement identitaire et décentralisateur, en essaimant du musée, projetait une situation de demande et attendait en retour un développement de la fréquentation. Vingt ans plus tard, la dissémination d'établissements patrimoniaux n'a accouché que d'un musée à la fréquentation suspecte ; quand le bourgeois collectionneur se surprend à côtoyer un petit-bourgeois à la bonne volonté culturelle.

Léchec réel de la démocratisation de l'institution, qui n'était que celui de politiques ministérielles volontaristes, allait justifier l'interruption de la modernisation des musées, la fin de leur mise en conformité avec les nouvelles formes du savoir et de la création.

Les projets scientifiques et culturels inscrivent brutalement la fin d'une ambition. Ils tracent des limites et réaffirment la volonté de l'État d'encadrer les musées, et tout particulièrement les musées de province. L'apparition de ce document est un indice parmi d'autres de la mise entre parenthèses de la capacité créatrice de la société, de sa capacité à perturber l'institution. La relation entre le monde, ses objets et l'homme - l'objet du musée -, se transforme en relation obsessionnelle à la cohérence, en soumission à la rigueur comme idéologie.

Le musée, par nature déséquilibré, saoul d'objets, se voue dorénavant à la stabilité et à la sobriété.

\section{Petite critique du langage : quand le projet est tout un programme}

Nous n'oserons pas dans ce texte même évoquer les termes de scientifique et culturel. Nous nous arrêterons juste sur le choix des vocables projet et programme, expressions volontairement vagues, un certain temps confondus par les concepteurs du document cadre des musées de France. Dans la mesure où ces mots, si ordinaires, constituent l'intitulé premier, de quelle logique relèvent-ils ?

Le « projet ", littéralement une mise en avant, n'aurait-il pas été retenu pour son caractère opaque ? Cet objet érigé face à nous fait figure de paravent. Autrement dit, il a pour fonction de brouiller la réalité. On ne peut écarter dans ce cas l'hypothèse d'une institution désormais intégrée au spectaculaire mensonger, placée au devant de la scène culturelle dans une forme accomplie de la société du spectacle.

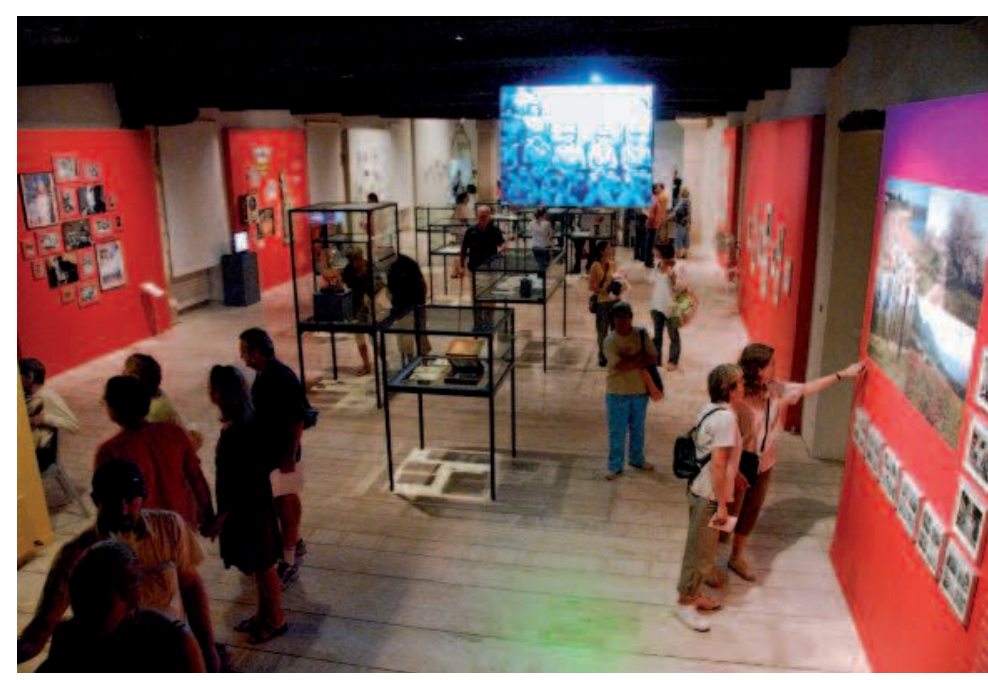

La salle d'exposition Durville ๑) Patrice Josserand

Quant au vocable " programme ", qui était si peu en usage avant le XIXe siècle, il s'impose au moment de la Révolution française. Drôle de concordance qui associe l'origine du mot et les textes fondateurs de l'abbé Grégoire. La Révolution française a souhaité unir le musée et le progrès pour que se tisse une chaîne ininterrompue entre l'action des anciens, nos gestes présents et l'avenir.

Programmer, c'est-à-dire écrire à l'avance, devient une suite d'opérations qui s'appuient sur la connaissance du passé pour légitimer les actes en cours. En héritant des mots des anciens, en fructifiant leur patrimoine, le réalisable et son double, le probable, se conçoivent. Vouloir réaliser un programme, c'est soumettre la pensée à la totalité comme sujet. La connaissance encyclopédique ne craint pas d'appréhender l'ensemble des connaissances afférentes à un sujet. Le projet assume tout, les généralités que le vocabulaire et ses fausses neutralités promettent. Le langage qui s'impose dans cet objet au statut juridique incertain et velléitaire adopte le ton administratif de «l'efficacité », du « rationnel » et du «possible». Pourtant, il comprend dans chacun de ses mots, dans sa structure (du général au particulier), dans sa rédaction, toute une conception de l'institution culturelle moderne. Le découpage "fonctionnel » du document préserve l'institution du choix, des relations, de l'analyse critique, de la déconstruction, toutes choses qui s'opposent à l'énumération, à l'accumulation, à l'inconscient et à l'a priori...

Il rappelle à l'ordre ; injonctions, obligations disent sèchement la nécessité de dissiper le malentendu des années 1980 et de celles qui ont suivi. On ne peut accepter la moindre imprécision. Le document contrôle et rejette les discours d'apparence indécise. 
L'ordre de cheminement dans le texte s'apparente à un questionnaire fermé, excluant toute échappée littéraire, poétique et artistique, même la plus anodine, qui risquerait d'attenter à la conformité du discours. Le PSC a une essence cachée ; contradictoirement obtus et sans limites, il offre la possibilité d'un document fleuve, regroupant plans, inventaires, textes, statistiques... Il accumule sous une forme digitale, les fonds, les archives du musée pour mieux le submerger, pour nous enivrer. Comme si les défilés avaient quelques vertus pour annoncer des victoires futures.

\section{Le PSC : un document bien tempéré ?}

Le problème du PSC réside dans sa perspective et c'est d'où nous le voyons qu'il prend forme, comme configuration pratique et idéologique. Le PSC est une structure construite, mieux même une préconstruction, une conception du musée qui nous est prodiguée. Le musée apparaîtrait là, circonscrit et complet, accompli, fort de l'expérience des générations précédentes de conservateurs, établi par les textes et les décrets.

Alors qu'elle se voit mûre et assurée, l'institution cache son infortune. Elle s'égare déboussolée dans un paysage culturel étrange et dévasté, conséquence de l'échec de la démocratisation culturelle et de nombreuses démissions empiriques. Peut-être parce que les musées et leurs conservateurs se sont tenus à l'écart des Sciences humaines, qu'ils craignent plus que tout la sociologie et la philosophie, l'idéologie en acte dans le PSC mélange allègrement fonctionnalisme et formalisme, pensées qui partagent avec le positivisme du XIXe siècle de se donner pour neutre et d'asseoir le pouvoir du technicien.

Le processus historique consiste à transformer le conservateur en ingénieur, à le condamner à la rédaction de notices, de dossiers de presse et d'actes administratifs. On lui dénie toute intention politique. Le PSC transforme en absolu la visite de chantier, le calcul des mètres carrés et le coût de fonctionnement ; vérités relatives mais simples portées techniques.

Le musée s'examine alors comme une succession de faits hiérarchisés, pareils à des données stratifiées dont la mise en relation est inconséquente puisque jamais politique et conceptuelle. Les faits ne parlent jamais par eux-mêmes, et, ici on se condamne à feindre lignorance des enjeux par de simples confirmations techniques. La place démesurée accordée aux études techniques diverses, la survalorisation du rôle du programmiste l'attestent. Le PSC se présente benoîtement en instrument de mesure dont les nor-

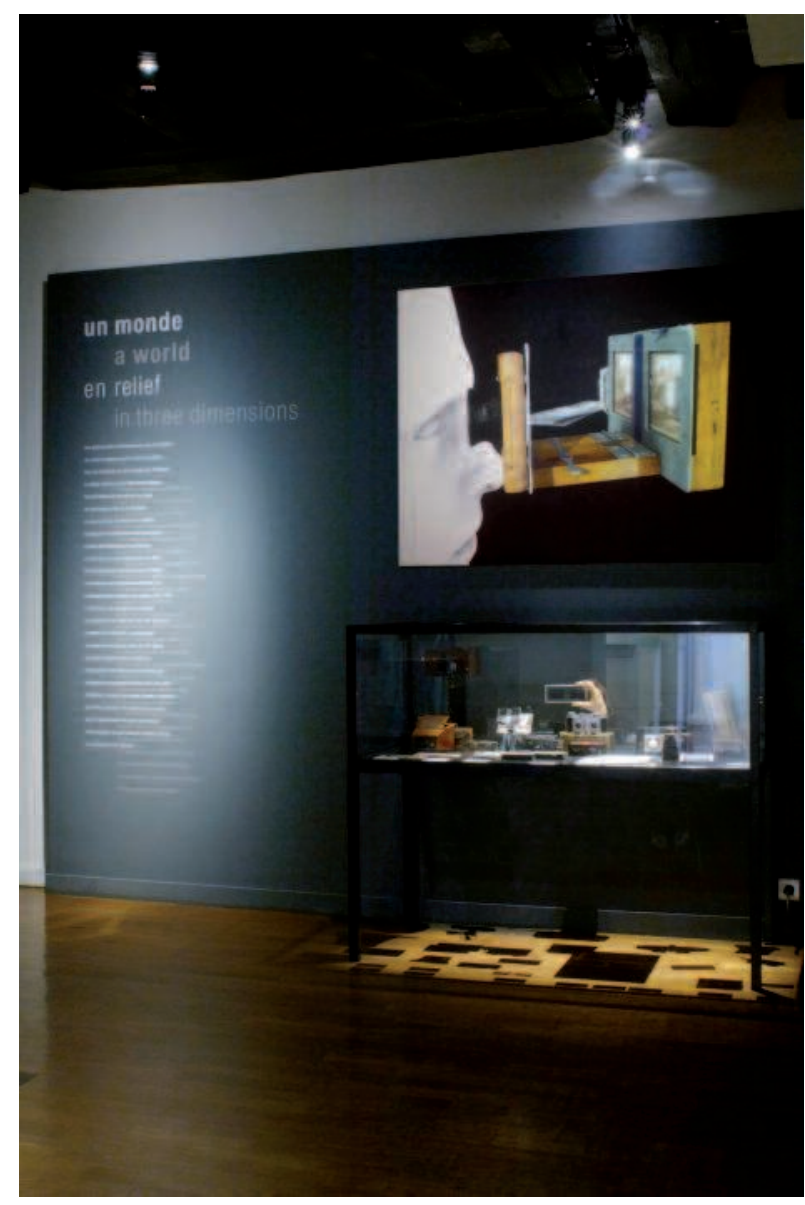

La salle consacrée aux photographies en relief (๑) Sylvain Charles

mes sont établies, faut-il le répéter par des agents de l'État, dans une optique de diminution du nombre de musées, considéré comme trop important, et de régulation de leur fonctionnement, réprimandé parce que jugé trop onéreux. Les opérations nécessaires à l'élaboration de ce qui est, il faut en convenir, un pensum, n'auraient d'autre but que d'évaluer la pratique muséographique et ses coûts. Or, de l'élaboration des multiples questionnaires (publics, tutelles, personnel...) et leur décodage jusqu'à l'analyse prévisionnelle du fonctionnement, l'ensemble de ces documents statistiques sont autant de théories en acte. Ils valident le musée en tant qu'entreprise culturelle. Ils concluent aux exigences de la concurrence entre musées. Consciemment ou inconsciemment, remplir ces grilles, accepter l'exercice technique, conduit à admettre le bien-fondé d'une théorie implicite d'un modèle d'organisation et ses présupposés.

Parce qu'il est un objet sans distance, le PSC établit un dispositif, de fait une prescription, une tentative de remise en ordre du musée dans la société actuelle, sans contestation de ses fondements inégalitaires. 


\section{Le musée Nicéphore Niépce}

Fondé en 1974 à Chalon-sur-Saône, ville de naissance de linventeur de la photographie, le musée Nicéphore Niépce a constitué en près de trente cinq ans d'existence l'une des collections photographiques les plus originales en Europe. Des premières héliographies de Nicéphore Niépce aux technologies numériques, cette collection de plus de trois millions d'images raconte les multiples histoires de la photographie dans son aventure esthétique et documentaire, mais aussi dans ses usages populaires et commerciaux.

Le musée se donne pour mission d'établir un rapport entre les inventeurs, les pionniers et les créateurs d'aujourd'hui et tourne sa réflexion vers l'analyse de l'image dans une acception plus globale : ses applications, ses effets, ses codes. Des dispositifs interactifs et des films pédagogiques visent à replacer le public au centre du discours. Le spectateur manipule virtuellement des appareils, plonge au cœur des expériences de Nicéphore Niépce, se situe dans les différents univers intimes, commerciaux, professionnels des pratiques photographiques. Les pièces muséographiques utilisant les nouvelles technologies de l'image, le site Internet (www.museeniepce.com) et la banque de données en ligne (www.dcimixer.com) favorisent l'accès de tous aux collections. Des projets éducatifs, des conférences, des stages nationaux ou internationaux inscrivent le musée dans son rôle formateur.

Lieu de visite, de conservation et d'exposition, le musée Nicéphore Niépce est également grâce à son laboratoire photographique un centre de production d'images contemporaines et de création artistique

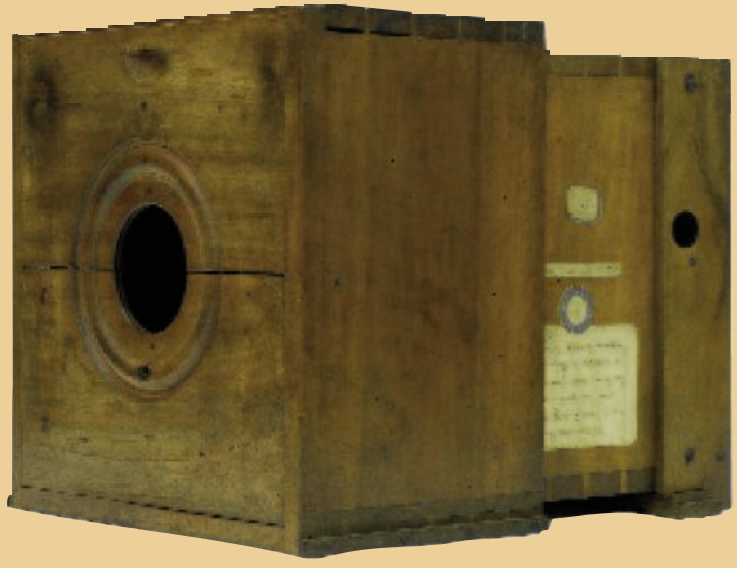

Appareil utilisé par Nicéphore Niépce vers 1820, appareil « de la découverte» ๑) Musée Nicéphore Niépce, ville de Chalon-sur-Saône

reconnu. Régulièrement cité dans les revues spécialisées comme un des 50 meilleurs musées de France (cette année 39e au classement des musées français), il sait conjuguer le respect de la conservation à l'étude précise des fonds conservés et à la présentation renouvelée de ses collections (plus de 60000 photographies ou objets des collections numérisés, une base de données riche de près de 140000 références et un plan de reconditionnement et de restauration des collections en cours depuis 2003).

\section{Musée Nicéphore Niépce \\ 28 quai des Messageries \\ 71100 Chalon-sur-Saône \\ téléphone + 33385484198 \\ contact@museeniepce.com \\ www.museeniepce.com}

En s'abritant derrière un vocabulaire fonctionnaliste, ce texte-cadre entérine la marchandisation du musée et son passage dans l'échange généralisé de la marchandise. Il favorise l'accélération du renouvellement des produits. L'exposition voit sa fonction se métamorphoser. Le stock, ce que l'on appelait autrefois la collection, inspire et redéfinit les critères de l'échelle des valeurs des produits. Le marché de l'art, qui englobe l'intégralité des objets, actionne des mécanismes efficaces qui poussent à transformer en biens de prestige des objets autrefois méprisés. Il est le lieu spécifique et primordial à l'extension du domaine des biens rares. La technique des échanges pour imposer ces nouveaux objets a donc besoin d'une communauté d'intérêts entre marchands, critiques, conservateurs et collectionneurs.
Le musée s'impose là déterminant dans la stratégie du désir et les conservateurs s'y agitent comme des spécialistes de l'objet, de son obsolescence ou de sa valorisation.

Dans cette perspective, on feint de s'étonner d'une apparente contradiction qui se manifesterait entre la réaffirmation «morale » de l'inventaire par l'État («fonds inaliénables »), et la soumission au court terme, objet de toutes les séductions, de tous les soutiens du marché et de ses mécènes. Ce ne sont en fait que les deux faces du marché qui manœuvrent face à la détérioration rapide des objets en se ressourçant dans le réservoir sans fond des musées. 


\section{Pas de code mais des principes}

\section{Le PSC au musée Nicéphore Niépce}

Le PSC exsude de tous ses pores le bon sens. Il s'attache à la statique et rejette la dynamique de l'établissement. Il recherche une matière et une forme qui seraient l'essence quand nous avons souhaité nous attacher aux forces en mouvement, quand nous réclamions une attitude où la puissance et l'audace pénétreraient dans l’inconnu.

Soumis à l'obligation de ce document, nous avons abordé l'exercice en prenant pour principe, ce qui était vrai, d'ignorer absolument le sujet même du musée, la photographie et ses propriétés caractéristiques. Une hypothèse de travail n'a pas besoin d'être vraie... Il fallait penser contre l'a priori, cela voulait dire mettre à jour les véritables raisons déterminant l'agir et la nature de notre action. Nos textes premiers se voulaient introspectifs et exprimaient le doute sur notre faculté de discerner avec clarté les enjeux, autrement complexes, dont procède la photographie.

Le PSC, comme document imposé, se révélera alors l'occasion d'exposer nos intentions, nos limites, nos contradictions. L'étape la plus difficile dans ce genre d'exercice consiste à refuser la posture divine. Bâtir un musée, ou le redéfinir, ne se résume pas à un geste d'ingénieur, mais il ne fait pas non plus du conservateur une figure tragique, un héros faustien. Au contraire, se construire avec le politique impose la lucidité indispensable à la compréhension des contradictions. Exercer la fonction de conservateur de musée commande en préalable à celui-ci de se situer dans un système des relations objectives dans lesquelles des groupes sociaux divers et antagonistes peuvent (doivent ?) se trouver intégrés dans le même lieu par une pratique foncièrement identique. L'architecture, les parcours, les scénographies, bref la morphologie du musée annonce les intentions culturelles, les déclare ou au pire les avoue.

Exposer c'est choisir et ne plus craindre de favoriser. La muséographie s'éloigne de sa logique interne (concurrence entre musées, stratégies de carrière, assujettissement au pouvoir...) et rejoint la sphère du refus et du combat qui est, aussi, la région de l'offre et de la proposition.

L'élaboration du principe explicatif du fonctionnement du musée Nicéphore Niépce intervient avec l'appréhension de la logique objective de l'organisation, établie contre l'expérience immédiate du visiteur, de

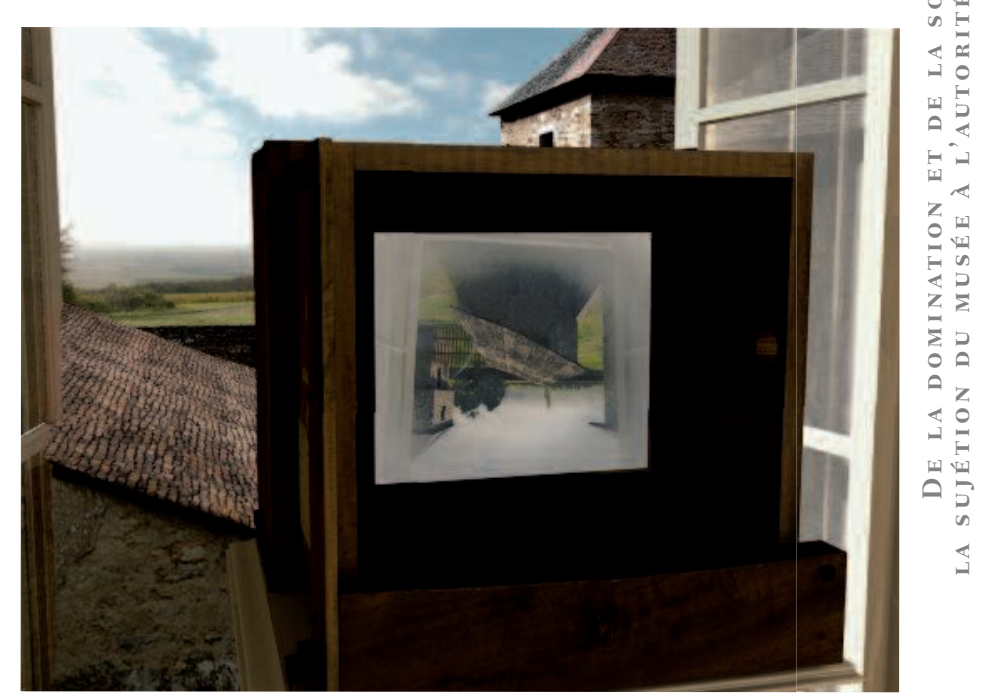

Extrait du film Sensible à la lumière écrit par Michel Frizot et réalisé par Jean-Michel Sanchez ๑) Musée Nicéphore Niépce, ville de Chalon-sur-Saône

l'historien d'art, du technologue, du collectionneur. C'est ainsi que nous sommes passés de la photographie au photographique et avons envisagé avec d'autres la photologie (Michel Frizot). Par pur raisonnement dialectique, en refusant la logique castratrice du PSC, nous avons abouti à définir les préceptes du musée Nicéphore Niépce. Constat paradoxal pour un musée de la photographie, il fallait accepter l'idée que ce que l'on voit n'est pas ce que l'on voit ! La mission dévolue à l'équipe muséale dans l'ensemble de ses tâches intellectuelles et opératoires devait rendre visible ce qui se révélait opaque, rendre accessible le complexe et ainsi créer des situations d'interrogations et de rupture avec le sens commun.

De cette expérience collective ont ainsi émergé des fondements clairs, suffisamment étayés pour que l'on puisse les considérer comme fondateurs :

- $1^{\text {er }}$ principe : le premier est de parler du premier, Nicéphore Niépce.

- 2e principe : rendre compte du mode de production de l'image mécanique.

- $3^{\mathrm{e}}$ principe : la photographie est un objet qui résiste.

- $4^{\mathrm{e}}$ principe : c'est dans un périmètre circonscrit entre quatre éléments que la photographie se constitue ; le preneur de vue, l'appareil ou le dispositif de capture, le sujet photographié et son devenir forment les points cardinaux de l'espace photographique.

- 5e principe : la visite du musée est toujours décevante, car pré-vue.

- $6^{\mathrm{e}}$ principe : la collection est aussi un stock et un avatar numériques. 


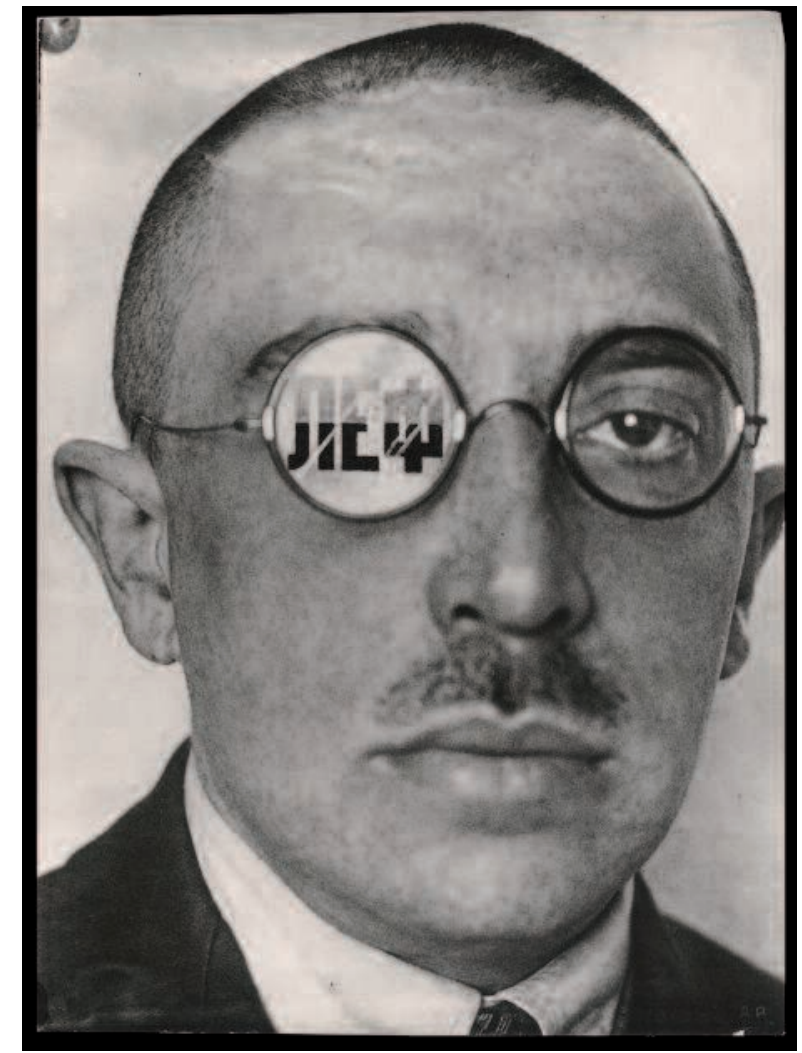

Alexandre Rodtchenko, Portrait d'O.-M. Brik (photomontage pour la revue LEF), 1924, épreuve au gélatino-bromure $d^{\prime}$ 'argent ๑) Musée Nicéphore Niépce, ville de Chalon-sur-Saône

- $7 \mathrm{e}$ principe : le photographique n'est pas réductible aux aléas du marché de la photographie.

- 8e principe : les derniers seront les premiers. Ce musée a une histoire.

C'est seulement après avoir déterminé les ressorts et les pièges de la réorganisation du musée que nous avons trouvé un passage de l'autre côté, vers une autre légitimité, dans le retour à un propos muséographique faisant de l'objet le révélateur d'un champ de forces antagoniques.

\section{Le PSC, triomphe du sens commun}

Le PSC, cette réduction «fonctionnaliste » du musée, nous apparaît, somme toute, comme une forme élaborée de recul démocratique. Il évacue le politique et nie son incapacité à remettre les missions du musée dans le système complet des relations dans lesquelles et par lesquelles elles s'accomplissent. Le corpus de chapitres, de sous-chapitres, de paragraphes, de questions, d'études, d'annexes, de plans, de statistiques garantissent l'influence et l'autorité de tutelles dépassées ou complices (Direction des Musées de France, Direction régionale des Affaires culturelles...). Ce texte, sous les contours modestes d'un vade-mecum, d'un manuel ou d'un guide constitue un code dont le corpus explicite commande et appelle à la soumission face à la «modernité ».

Ce n'est pas le lieu, ou peut-être est-il encore trop tôt, pour analyser les conséquences entre la généralisation de ce document et l'état des institutions muséales françaises ${ }^{(1)}$. Ce texte légalise les nouveaux états de fait. Il institue, grâce à une bureaucratie dévouée, une redéfinition du musée qui fait de ce «matériau » un objet nouveau, un hybride particulier entre la banque de données, une annexe du Louvre des Antiquaires..., une agence de cotations des valeurs esthétiques.

\section{Note}

(1) Il faudra pourtant que l'on s'interroge sur la portée du PSC et la situation des musées de société et d'histoire, des écomusées, des centres d'interprétation... On ne s'étonnera plus de la perpétuelle remise en question de l'inaliénabilité des collections. Le PSC fait l'impasse sur ce que l'on voit depuis peu, la privatisation partielle de l'institution muséographique : les grands établissements essaimant des filiales dans les nouveaux pays sans culture muséographique dont le but est de s’insérer dans un système normalisé de références culturelles identiques. 